\title{
847 PHARMACOKINETICS AND PHARMACODYNAMICS OF GS-3583 IN CYNOMOLGUS MONKEYS
}

Michelle Kuhne*, Hamlet Chu, Christopher Clarke, Brian Carr, Manuel Baca, Magdeleine Hung, Mark Nagel, Alexandre Ambrogelly, Nishanathan Rajakumaraswamy. Gilead Sciences, San Francisco, USA

Background The ligand for the receptor tyrosine kinase FMSlike tyrosine kinase 3 (FLT3) plays an importantrole in hematopoiesis. FLT3 signaling is required for the differentiation andexpansion of dendritic cells. In the context of cancer immunity, the conventional dendritic cellsubtype 1 (cDC1) are required for the generation of tumor-specific $\mathrm{T}$ cell responses in mousepreclinical models. In human tumors $\mathrm{cDC} 1$ are often underrepresented in thetumor microenvironment, supporting the hypothesis that therapeutically increasing their number via FLT3 pathway stimulation has the potential to promote $\mathrm{T}$ cellmediated anti-tumor activity.

Methods GS-3583 is a fusion protein composed of the extracellular domain of human FLT3 ligand(FLT3L) combined with a modified fragment crystallizable $(\mathrm{Fc})$ region of human IgG4. GS-3583was designed to induce cDC1 expansion and subsequently promote tumor-reactive $\mathrm{T}$ cell priming, activation and recruitment into the tumor microenvironment. The pharmacokinetics (PK) and pharmacodynamics (PD) of GS-3583 has been characterized in a 4-week repeat dose GLP study in cynomolgus monkeys at doses ranging from 0.3 to $10 \mathrm{mg} / \mathrm{kg}$ GS3583 was given as an intravenous injection.

Results Immunophenotyping analysis of peripheral blood cells from GS-3583 treated monkeys demonstrated a non-dosedependent expansion of $\mathrm{CDC} 1$ and $\mathrm{CDC} 2$ populations. The peak expansion for $\mathrm{cDC} 1$ and $\mathrm{cDC} 2$ occurred at Day 8 to Day 15. At peak, there was a 160 -fold relative increase in cDC1 and 150-fold increase in $\mathrm{CDC} 2$ at the highest dose tested. There were dose-dependent increases in the exposure (AUC and Cmax) of GS-3583. GS-3583 was well-tolerated with no mortality or adverse clinical signs.

Conclusions The administration of GS-3583 leads to increases in $\mathrm{CDC} 1$ and $\mathrm{CDC} 2$ populations. It was well tolerated at the maximal dose tested with no adverse clinical signs. Further clinical development of GS-3583 is warranted.

http://dx.doi.org/10.1136/jitc-2021-SITC2021.847 